\title{
RESEARCH
}

Open Access

\section{Preliminary results of Preserflo Microshunt versus Preserflo Microshunt and Ologen implantation}

Iraklis Vastardis ${ }^{1^{*}}$ (D), Sofia Fili ${ }^{1}$, Georgios Perdikakis ${ }^{1}$, Kalliopi Kontopoulou' ${ }^{1}$ Miltos Balidis ${ }^{2}$, Zisis Gatzioufas ${ }^{3}$ and Markus Kohlhaas ${ }^{1}$

\begin{abstract}
Purpose: To report preliminary 6-month results on the use of the Preserflo Microshunt implant with and without Ologen in 50 pseudophakic eyes with moderate to advanced primary open-angle glaucoma (POAG).

Methods: Fifty pseudophakic eyes underwent ab externo Preserflo Microshunt implantation. Data was gathered retrospectively and two groups were then created, group A with application of MMC $0.2 \mathrm{mg} / \mathrm{ml}$ and group B with MMC $0.2 \mathrm{mg} / \mathrm{ml}$ and Ologen collagen matrix (OCM) implantation. Absolute success was regarded as the percentage of eyes achieving: a) $5 \leq$ intraocular pressure (IOP) $\leq 13 \mathrm{mmHg}$, b) $5 \leq 1 \mathrm{OP} \leq 16 \mathrm{mmHg}$, and c) $5 \leq 1 \mathrm{OP} \leq 21 \mathrm{mmHg}$ without additional medication or surgery and qualified success was regarded as the percentage of eyes achieving a) IOP $\leq 13 \mathrm{mmHg}$, b) IOP $\leq 16 \mathrm{mmHg}$, and c) IOP $\leq 21 \mathrm{mmHg}$ with or without medication. Evaluation was performed using a log-rank Kaplan-Meier test. A scatterplot analysis presented the treatment effect data of all eyes with a minimum of 20\% IOP reduction per case. Failure was defined as requiring additional surgery, IOP greater than $21 \mathrm{mmHg}$ with or without medication and failure to reach 20\% IOP reduction.

Results: Mean postoperative IOP was significantly lower in both groups. IOP decreased by $49.06 \%$ in group A and by $53.01 \%$ in group B at 6 months $(P<0.88)$, respectively. Medication use was lower in both groups (Wilcoxon test, $P<0.001$ ). The absolute and qualified success rates were not statistically significant between the groups (all $P>$ 0.05). Cumulative IOP results per case were not statistically different in group A compared with group B. One revision surgery in group A (4\% failure rate) and three in group B (12\% failure rate) were performed.

Conclusions: Both groups showed equal results in terms of cumulative and mean IOP reduction, medication reduction as well as in absolute and qualified success rates. No significant difference was found in any parameters tested between Preserflo Microshunt with MMC $0.2 \mathrm{mg} / \mathrm{ml}$ and with or without OCM implantation at 6 months. Long-term follow-up is required to further evaluate this data.
\end{abstract}

Keywords: Micro invasive glaucoma surgery, Ab externo surgery, Refractory POAG, SIBS polymer, Mitomycin C

\footnotetext{
* Correspondence: vastardis.iraklis@gmail.com

'Department of Ophthalmology, St. Johannes Academic Hospital Dortmund, Dortmund, Germany

Full list of author information is available at the end of the article
}

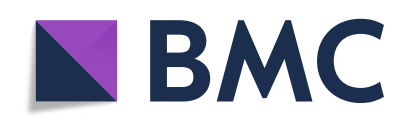

(- The Author(s). 2021 Open Access This article is licensed under a Creative Commons Attribution 4.0 International License, which permits use, sharing, adaptation, distribution and reproduction in any medium or format, as long as you give appropriate credit to the original author(s) and the source, provide a link to the Creative Commons licence, and indicate if changes were made. The images or other third party material in this article are included in the article's Creative Commons licence, unless indicated otherwise in a credit line to the material. If material is not included in the article's Creative Commons licence and your intended use is not permitted by statutory regulation or exceeds the permitted use, you will need to obtain permission directly from the copyright holder. To view a copy of this licence, visit http://creativecommons.org/licenses/by/4.0/. The Creative Commons Public Domain Dedication waiver (http://creativecommons.org/publicdomain/zero/1.0/) applies to the data made available in this article, unless otherwise stated in a credit line to the data. 


\section{Introduction}

Glaucoma is still responsible for irreversible blindness globally [1]. Patient numbers around the world are rising and projected to reach 111.8 million soon [2]. Visual field deterioration in such patients can be prevented by lowering the intraocular pressure (IOP) which is achieved either through medication, laser treatment or surgery [3-5]. Trabeculectomy is widely performed and still regarded as the gold standard treatment. Although the method itself has been rigorously improved over the years [6], its use among surgeons is declining in favor of newer micro invasive glaucoma surgery (MIGS) devices [7]. Surgeons appear to be favoring such alternatives due to their superior safety and efficacy profiles [8]. These glaucoma implants have been introduced as a minimally invasive surgical option to prevent further glaucoma deterioration. These devices provide an outflow pathway of the aqueous humor from the anterior chamber of the eye into the posterior subtenonal space, and thus reduce IOP by mimicking the mechanism of trabeculectomy, while simultaneously conferring a standardized IOP outcome and an enhanced safety profile [9-11]. Such a device is the Preserflo ${ }^{\text {tm }}$ Microshunt (Santen, Osaka, Japan) implant formerly known as the InnFocus Microshunt (Santen, Osaka, Japan). It is a minimally invasive glaucoma drainage device that forms a posterior filtration bleb under the conjunctiva and Tenon's capsule. Preserflo Microshunt consists of a polymer, the poly-styrene-block-isobutylene-block-styrene (SIBS), a stretchy material, that provokes minimal inflammation and encapsulation in comparison to other materials used in glaucoma surgery such as silicone rubber and polypropylene [12, 13]. SIBS technology was introduced in 1999 through TAXUS ${ }^{\circ}$ (Boston Scientific's, Natick, Massachusetts, USA) as a coronary stent in surgical treatment and prevention of arterial restenosis [14]. Today, as a glaucoma microimplant and through its unique thermo-formable features it confers multiple advantages over traditional thermoset materials such as silicone rubber [12, 13]. There are multiple clinical trials evaluating its efficacy which are currently underway in Europe, USA, Singapore, Japan and the Dominican Republic. This case series study aims to not only report preliminary 6-month results on the use of the Preserflo Microshunt in terms of mean IOP and medication reduction, but also to compare two different surgical approaches: implantation of the Preserflo Microshunt with mitomycin $C(M M C, 0.2 \mathrm{mg} / \mathrm{ml})$ treatment and implantation of the Preserflo Microshunt with MMC $0.2 \mathrm{mg} / \mathrm{ml}$ treatment and additional Ologen collagen matrix (OCM) implantation. OCM is a biodegradable porous collagen-glycosaminoglycan copolymer matrix implant claiming wound modulation among connective and epithelial tissues. It also serves as a spacer or barrier between the sclera surface and conjunctiva [15]. Although its true benefit in glaucoma surgery is still being debated [16, 17], OCM is currently being widely utilized extending beyond glaucoma surgery [18] to other ocular pathologies, for instance in pterygium excision where it modulates the wound healing of the conjunctiva and serves as an inhibitor of recurrence [19]. In this cohort, both the late wound healing modulation of the bleb and the benefit of space creation between the Preserflo Microshunt and the Tenon's capsule were evaluated. Our null hypothesis was that the IOP should be better in the eyes that underwent additional OCM implantation in comparison with the eyes that did not, by reducing the fibrotic reaction in the bleb area. The alternative hypothesis was that the IOP was equally lowered through the Preserflo Microshunt implantation with MMC $0.2 \mathrm{mg} / \mathrm{ml}$ regardless of the additional OCM implantation. If the OCM implantation indeed reduces the fibrotic reaction in the bleb area either through its properties or by acting as a barrier or spacer, then a reduction of revision surgery, needling interventions and additional use of antimetabolites or chemotherapeutical agents such as 5fluorouracil (5-FU) should be reported.

\section{Material and methods}

Fifty Caucasian patients and fifty eyes with treatment refractory primary open-angle glaucoma (POAG) were operated in 2020. Twenty-five eyes (group A) underwent a Preserflo Microshunt implantation with MMC $(0.2 \mathrm{mg} / \mathrm{ml})$, while the remaining 25 eyes (group B) underwent the same surgery with additional OCM implantation (Aeon Astron Europe BV, Leiden, The Netherlands). All surgeries were carried out under general anesthesia, performed by five Preserflo Microshunt certified surgeons, and took place in Saint Johannes Hospital, Ophthalmology Department, in Dortmund, Germany. All patients provided their written consent prior to surgery and the tenets of the Declaration of Helsinki were fully adhered to. The institution's ethical committee approved this retrospective case series study. The primary patient selection criterion for this study was POAG refractory to medical treatment with prior uncomplicated cataract extraction. Inclusion and exclusion criteria are listed in Table 1. The baseline examination included a complete ophthalmic history, Humphrey visual field perimetry SITA Fast, endothelial microscopy (Tomey EM 4000, Tomey GmBH Technology and Vision, Nürnberg, Germany), Scheimpflug corneal 
Table 1 Inclusion and exclusion criteria of the study cohort Inclusion Criteria

Refractory to medical treatment POAG and moderate to advanced POAG according to HPA classification system

Prior uncomplicated cataract extraction, no prior history of glaucoma surgery or laser treatment

$A C D \geq 2.7 \mathrm{~mm}, \mathrm{ACA} \geq$ Schaffer $3-4^{\circ}$

\section{Exclusion Criteria}

Secondary glaucoma, ACG, OHT, PDG, PEXG, LTG, prior glaucoma surgery or laser treatment

Prior refractive surgery, PPV, phakic patients, corneal and retinal pathology, AMD, CME, last eye - monocular

No glaucoma medication or mono-therapy

POAG primary open-angle glaucoma; HPA Hodapp-Parrish-Anderson classification system; $A C D$ anterior chamber depth; $A C A$ anterior chamber angle; $A C G$ angle-closure glaucoma; $O H T$ ocular hypertension; $P D G$ pigmentary dispersion glaucoma; PEXG pseudoexfoliation glaucoma; LTG low-tension glaucoma; PPV pars plana vitrectomy; $A M D$ age-related macular degeneration; CME cystoid macular edema

tomography (Pentacam, OCULUS Optikgeräte GmbH, Germany), high-definition ocular coherence tomography (HD-OCT) optic disc and ganglion cell layer evaluation (Cirrus HD-OCT 500,Carl Zeiss Meditec AG, Germany), single measurement of calibrated Goldman applanation tonometry, Snellen visual acuity, slit-lamp anterior and posterior segment examination and gonioscopy with angle grading. Data was gathered again concurrently at the 1st day, 2nd week, 1st, 3rd, and 6th month including standard slit-lamp examination of the anterior and posterior segment, Goldman applanation tonometry, visual acuity testing and postoperative complications.

\section{Surgical technique}

For both groups, a 4-mm fornix based conjunctival peritomy was performed in the upper temporal or nasal quadrant in order to reveal the underlying sclera. Cauterization was performed under balanced salt solution (BSS) irrigation followed by scleral treatment with three $\mathrm{MMC}(0.2 \mathrm{mg} / \mathrm{ml})$ soaked sponges posteriorly for three minutes. An ink marking was then placed $3 \mathrm{~mm}$ posterior to limbus followed by creation of an initial pocket with a 1.2$\mathrm{mm}$ diamond blade. A 25-Gauge needle was then bent with the bevel up and inserted at a $90^{\circ}$ angle into the anterior chamber, exiting at the level of the trabecular meshwork. The device was then slid through the pocket and tunnel while the fins of the device were stabilized into the scleral pocket. The aqueous humor flow chamber was tested with a sponge via observation. Finally, the device was covered from the conjunctiva and Tenon's capsule, which in turn they were separately reattached with a 10-0 nylon running suture.
For group B exclusively, the $6 \mathrm{~mm}$ wide and $2 \mathrm{~mm}$ high OCM, was additionally placed posterior to the device's fin and then tucked beneath the Tenon's capsule and conjunctiva, prior to their reattachment with the 10-0 nylon running suture. Postoperative blebs were evaluated according to the Wuerzburg bleb classification score (WBCS) [20, 21]. It is based on a score that evaluates vascularization, corkscrew vessel and encapsulation but not the height of the bleb [22]. The postoperative medication in both groups was moxifloxacin three times daily and cycloplegics two times daily for the first week combined with preservative free corticosteroid eye drops instilled six times daily. After the first week, the antibiotics and cycloplegics were discontinued and the corticosteroid regimen was tapered by reducing one drop every week for the next 6 weeks.

\section{Statistical analysis}

The mean IOP and medication reduction as well as the corrected distance visual acuity (CDVA) were evaluated with a two-way analysis of variance (twoway ANOVA) and paired samples t-test. Medication reduction between baseline and 6 months was reported using Wilcoxon test. Homoscedasticity, assumption of homogeneity, outliers assumption and the distribution of data for two-way ANOVA were also tested. Efficacy was assessed with the KaplanMeier survival analysis in three different postoperative IOP goals between the groups and with two different success criteria. Absolute success was regarded as the percentage of eyes that achieved a) $5 \leq \mathrm{IOP} \leq 13 \mathrm{mmHg}, \quad$ b) $5 \leq \mathrm{IOP} \leq 16 \mathrm{mmHg}$, and c) $5 \leq \mathrm{IOP} \leq 21 \mathrm{mmHg}$ without additional medication or surgery and qualified success was regarded as the percentage of eyes that achieved a) IOP $\leq 13 \mathrm{mmHg}$, b) IOP $\leq 16 \mathrm{mmHg}$, and c) IOP $\leq 21 \mathrm{mmHg}$ with or without medication. We included a scatterplot analysis to present the treatment effect data of all eyes in this study that demonstrates the minimum 20\% IOP reduction requirement achieved in both groups. Failure was defined as additional surgery, IOP greater than $21 \mathrm{mmHg}$ with or without medication and failure to reach $20 \%$ IOP reduction. Cumulative IOP results per group were also evaluated and presented in percentages for IOP readings less or equal than 11,13 and $15 \mathrm{mmHg}$. Intraoperative and postoperative complications as well as additional surgery rates were also evaluated as percentages. Statistical analysis was performed with MedCalc ${ }^{\circ}$ 16.2.1 and IBM SPSS $^{\circ}$ version 22. Parametric or non-parametric tests were used according to distribution normality (Kolmogorov-Smirnov). We considered statistical significance as a $P$ value $<0.05$. 


\section{Results}

Thirty five cases were classified as advanced POAG (with a mean visual field mean deviation (MD) $20.50 \pm 6.38 \mathrm{~dB}$ ) and 15 cases as moderate (mean visual field $\mathrm{MD}-7.37 \pm 0.71 \mathrm{~dB}$ ) according to the Hodapp-Parrish-Anderson (HPA) classification system [23]. Demographics are presented in Table 2. All variables tested for the results presented between both groups A and B were comparable (Table 2). The mean postoperative IOP decreased significantly from baseline in both surgical groups. IOP in group A decreased from $23.52 \pm 5.78$ to $11.56 \pm 3.08 \mathrm{mmHg}$ (49.06\% mean IOP reduction) and from $26.04 \pm 8.76$ to $11.75 \pm 3.37 \mathrm{mmHg}$ (53.01\% mean IOP reduction) in group B by the 6th month (two-way ANOVA, $P<0.001$; Fig. 1). Medication decreased in both groups compared to baseline (Table 3). Medication use in group A decreased from $2.52 \pm 0.91$ to $0.04 \pm$ 0.20 (98.02\% reduction, a median reduction of 2.5 medications). Medication use in group B decreased from $2.58 \pm 0.82$ to $0.16 \pm 0.81$ (94.44\% reduction, a median reduction of 2.5 medications) by the 6th month (Wilcoxon test, $P<0.001$ ). The Levene's equality test was statistically significant $(P<0.001$, Table 4), and thus the data tested failed to meet the assumption of homogeneity and outliers assumption between the groups. Regarding the power of the samples, we found that the follow-up was the only statistically significant factor $(P<0.001)$ in terms of IOP reduction with partial eta squared $0.63(63 \%)$ and observed power of 1 . No other factor or pairwise factor was significant in terms of IOP reduction (Table 4). CDVA was stable in both groups (paired samples t-test, $P=0.95$, from baseline $0.64 \pm 0.24$ to the 6th month $0.66 \pm 0.21$ Snellen and $P=0.33$, from baseline $0.58 \pm 0.27$ to the 6th month $0.61 \pm 1.93$ Snellen, in groups A and B, respectively). Absolute success rates in group A accounted for a) $48 \%$, b) $64 \%$ and c) $68 \%$ in comparison to group B a) $45.8 \%$, b) $45.8 \%$ and c) $58.3 \%$ at 6 months (Figs. 2, 3 and 4 ). Qualified success rate in group A accounted for a) $68 \%$, b) $88 \%$ and c) $92 \%$ in comparison to group B a) $70.8 \%$, b) $91.8 \%$ and c) $95.8 \%$ at 6 months (Figs. 5, 6 and 7). Log-rank Kaplan-Meier showed no statistical differences between the groups and parameters tested $(P<0.68, P<0.22, P<0.48$ absolute success and $P=0.38, P<0.31$ and $P<0.58$ qualified success, group A and group B, respectively). The scatterplot analysis verified those results (Fig. 8) by showing a $20 \%$ IOP reduction and an overall IOP reduction per

Table 2 Demographics of study participants

\begin{tabular}{|c|c|c|c|}
\hline Demographics & $\mathbf{n}$ & & \\
\hline No. of patients & 50 & & \\
\hline No. of female patients & 33 & & \\
\hline Age (years) & $76.13 \pm 10.08$ & & \\
\hline No. of male patients & 17 & & \\
\hline Age (years) & $79.00 \pm 7.94$ & & \\
\hline No. of eyes operated total (POAG refractory to medical treatment) & 50 & & \\
\hline Baseline MD (dB) total & $-13.31 \pm 7.93$ & & \\
\hline Advanced POAG, Baseline MD (dB) $(n=35)$ & $-20.50 \pm 6.38$ & & \\
\hline \multirow[t]{2}{*}{ Moderate POAG, Baseline MD (dB) $(n=15)$} & $-7.37 \pm 0.71$ & & \\
\hline & $\begin{array}{l}\text { Group A } \\
\text { (n) }\end{array}$ & Group B & $\begin{array}{l}\text { Intergroup variable comparison } \\
\text { t-test, } P<0.05(95 \% \mathrm{Cl})\end{array}$ \\
\hline No. of eyes operated total & 25 & 25 & N.A \\
\hline No. of pseudophakic eyes & 25 & 25 & N.A \\
\hline Age (years) & $77.58 \pm 7.62$ & $75.64 \pm 12.61$ & $0.88(-6.15$ to 5.32$)$ \\
\hline Baseline MD (dB) & $-13.15 \pm 8.53$ & $-13.47 \pm 7.44$ & $0.84(-4.57$ to 3.78$)$ \\
\hline Baseline average RNFL thickness ( $\mu \mathrm{m})$ & $65.04 \pm 8.03$ & $64.54 \pm 10.67$ & $0.52(-3.63$ to 6.78$)$ \\
\hline Baseline IOP (mmHg) & $23.52 \pm 5.78$ & $26.04 \pm 8.76$ & $0.26(-1.93$ to 6.77$)$ \\
\hline Baseline antiglaucoma agents & $2.52 \pm 0.91$ & $2.58 \pm 0.82$ & $0.36(-0.38$ to 0.98$)$ \\
\hline Baseline DCVA (Snellen) & $0.64 \pm 0.24$ & $0.58 \pm 0.27$ & $0.43(-0.23$ to 0.10$)$ \\
\hline
\end{tabular}

POAG primary open-angle glaucoma; IOP intraocular pressure; $M D$ mean deviation; $d B$ decibels; $R N F$ retinal nerve fiber layer; CDVA corrected distance visual acuity; $M M C$ mitomycin C; N.A not available

Group A: Preserflo Microshunt with MMC $0.2 \mathrm{mg} / \mathrm{ml}$

Group B: Preserflo Microshunt with MMC $0.2 \mathrm{mg} / \mathrm{ml}$ and Ologen 


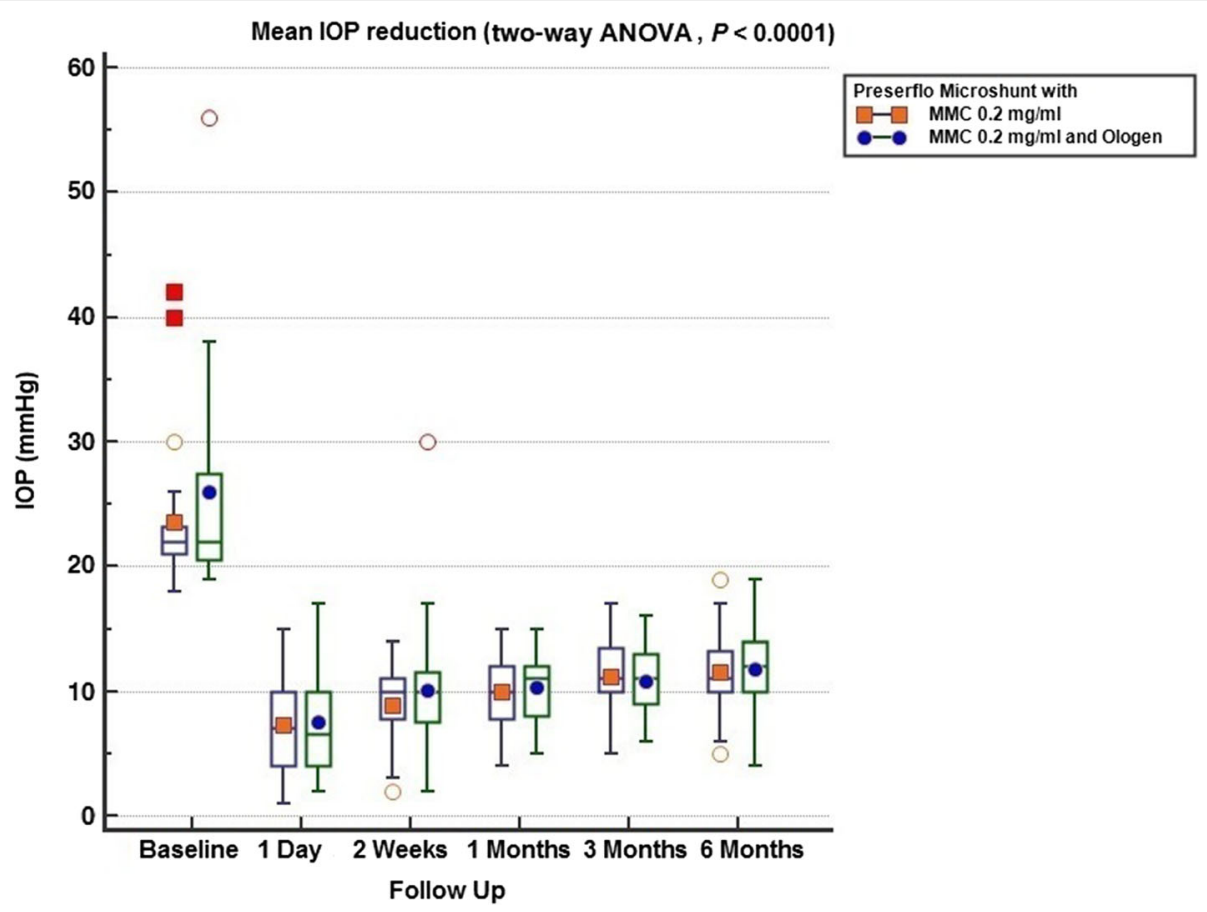

Fig. 1 Box and Whisker plot of the mean intraocular pressure (IOP) reduction between Preserflo Microshunt with MMC $0.2 \mathrm{mg} / \mathrm{ml}$ and Preserflo Microshunt with MMC $0.2 \mathrm{mg} / \mathrm{ml}$ and Ologen implantation (two-way ANOVA, $P<0.001$ )

case for all 50 eyes in both groups. The cumulative IOP results per group were not statistically different in group A compared to group B at 6 months (Fig. 9 and Table 4). We did not observe any severe intraoperative complications in either group. In the early postoperative follow-up, 12 eyes (24\%) presented with transient hypotony (IOP $<5 \mathrm{mmHg}$ ) for 1 week, in which 6 of them (50\%) presented a minor choroidal detachment in the retinal periphery which resolved within 14 days, while in 1 eye (2\%) the choroidal detachment persisted for over 1 month and spontaneously resolved after immediate discontinuation of the corticosteroid topical treatment. Twenty-six eyes (52\%) had a normal postoperative follow-up without any interventions or complications. Four revision surgeries due to bleb fibrosis were performed at 6 months and were considered failures (8\%). Three revision surgeries were

Table 3 Paired samples t-test comparison of the means in terms of medication in both groups

\begin{tabular}{|c|c|c|c|c|c|c|}
\hline Follow Up & Intervention & $\mathbf{n}$ & Mean \pm SD & Std. Error & 95\% Confidence interval & $P$ \\
\hline \multirow[t]{2}{*}{ Baseline } & Group A & 25 & $2.52 \pm 0.91$ & 0.19 & 2.10 to 2.89 & 0.70 \\
\hline & Group B & 25 & $2.58 \pm 0.82$ & 0.16 & 2.23 to 2.93 & \\
\hline \multirow[t]{2}{*}{1 Day } & Group A & 25 & $0.00 \pm 0.00$ & 0.00 & 0.00 to 0.00 & 0.00 \\
\hline & Group B & 25 & $0.00 \pm 0.00$ & 0.00 & 0.00 to 0.00 & \\
\hline \multirow[t]{2}{*}{2 Weeks } & Group A & 25 & $0.00 \pm 0.00$ & 0.00 & 0.00 to 0.00 & 0.00 \\
\hline & Group B & 25 & $0.00 \pm 0.00$ & 0.00 & 0.00 to 0.00 & \\
\hline \multirow[t]{2}{*}{1 Month } & Group A & 25 & $0.00 \pm 0.00$ & 0.00 & 0.00 to 0.00 & 0.00 \\
\hline & Group B & 25 & $0.00 \pm 0.00$ & 0.00 & 0.00 to 0.00 & \\
\hline \multirow[t]{2}{*}{3 Months } & Group A & 25 & $0.00 \pm 0.00$ & 0.00 & 0.00 to 0.00 & 0.00 \\
\hline & Group B & 25 & $0.00 \pm 0.00$ & 0.00 & 0.00 to 0.00 & \\
\hline \multirow[t]{2}{*}{6 Months } & Group A & 25 & $0.04 \pm 0.20$ & 0.04 & -0.04 to 0.12 & 0.47 \\
\hline & Group B & 25 & $0.16 \pm 0.66$ & 0.16 & -0.17 to 0.51 & \\
\hline
\end{tabular}


Table 4 Two-way ANOVA with tests of between-subjects effects for all variables tested, estimated marginal means and pairwise comparison for the IOP dependent variable and intergroup t-test comparison between Preserflo Microshunt with MMC $0.2 \mathrm{mg} / \mathrm{ml}$ and Preserflo Microshunt with MMC $0.2 \mathrm{mg} / \mathrm{ml}$ and Ologen implantation

\begin{tabular}{|c|c|c|c|c|c|c|c|}
\hline \multicolumn{8}{|l|}{ Two-way ANOVA } \\
\hline \multicolumn{8}{|c|}{ Levene's test for equality of error variances } \\
\hline $\mathbf{F}$ & \multicolumn{2}{|l|}{ DF 1} & \multicolumn{2}{|l|}{ DF 2} & \multicolumn{3}{|l|}{$P$} \\
\hline 3.9319 & \multicolumn{2}{|l|}{11} & \multicolumn{2}{|l|}{271} & \multicolumn{3}{|l|}{$<0.001$} \\
\hline \multicolumn{8}{|c|}{ Tests of between-subjects effects (all variables) } \\
\hline Source & Sum of squares & DF & Mean square & $\mathbf{F}$ & Partial eta squared & Observed power ${ }^{b}$ & $P$ \\
\hline Follow up & 9514.47 & 5 & 1902.89 & 98.70 & 0.639 & 1.0 & $<0.001$ \\
\hline Intervention & 36.31 & 1 & 36.31 & 1.88 & 0.06 & 0.27 & 0.17 \\
\hline Follow up*Intervention & 64.22 & 5 & 12.84 & 0.66 & 0.01 & 0.25 & 0.64 \\
\hline Residual & 5224.34 & 271 & 19.27 & & & & \\
\hline \multicolumn{8}{|c|}{ Estimated marginal means (IOP dependent) } \\
\hline \multicolumn{2}{|l|}{ Follow up } & $\mathbf{n}$ & \multicolumn{2}{|l|}{ Mean } & Std. Error & \multicolumn{2}{|c|}{ 95\% Confidence interval } \\
\hline \multicolumn{2}{|l|}{ Baseline } & 50 & \multicolumn{2}{|l|}{24.78} & 0.62 & \multicolumn{2}{|l|}{23.54 to 26.01} \\
\hline \multicolumn{2}{|l|}{1 Day } & 50 & \multicolumn{2}{|l|}{7.37} & 0.62 & \multicolumn{2}{|l|}{6.13 to 8.60} \\
\hline \multicolumn{2}{|l|}{2 Weeks } & 50 & \multicolumn{2}{|l|}{9.48} & 0.62 & \multicolumn{2}{|l|}{8.24 to 10.71} \\
\hline \multicolumn{2}{|l|}{1 Month } & 50 & \multicolumn{2}{|l|}{10.12} & 0.62 & 8.89 to 11.36 & \\
\hline 3 Months & & 50 & 10.98 & & 0.62 & 9.74 to 12.21 & \\
\hline 6 Months & & 50 & 12.18 & & 0.71 & 10.78 to 13.58 & \\
\hline Follow up & Intervention & $\mathbf{n}$ & Mean \pm SD & & Std. Error & t-test $(P)$ & 95\% Confidence interval \\
\hline Baseline & Group A & 25 & $23.52 \pm 5.88$ & & 0.87 & 0.26 & 21.79 to 25.24 \\
\hline & Group B & 25 & $26.04 \pm 8.76$ & & 0.89 & & 24.27 to 27.80 \\
\hline 1 Day & Group A & 25 & $7.24 \pm 3.47$ & & 0.87 & 0.76 & 5.51 to 8.96 \\
\hline & Group B & 25 & $7.50 \pm 4.23$ & & 0.89 & & 5.73 to 9.26 \\
\hline 2 Weeks & Group A & 25 & $8.88 \pm 3.08$ & & 0.87 & 0.34 & 7.15 to 10.60 \\
\hline & Group B & 25 & $10.08 \pm 5.48$ & & 0.89 & & 8.31 to 11.84 \\
\hline 1 Month & Group A & 25 & $9.92 \pm 3.25$ & & 0.87 & 0.49 & 8.19 to 11.64 \\
\hline & Group B & 25 & $10.33 \pm 2.53$ & & 0.89 & & 8.56 to 12.09 \\
\hline 3 Months & Group A & 25 & $11.20 \pm 2.71$ & & 0.89 & 0.63 & 9.44 to 12.97 \\
\hline & Group B & 25 & $10.76 \pm 2.78$ & & 0.87 & & 9.03 to 12.48 \\
\hline 6 Months & Group A & 25 & $12.00 \pm 3.13$ & & 0.63 & 0.89 & 10.01 to 13.98 \\
\hline & Group B & 25 & $12.36 \pm 3.37$ & & 0.67 & & 10.38 to 14.35 \\
\hline Pairwise comparisons & (IOP dependent) & & Mean differenc & & Std. Error & $P^{\mathrm{a}}$ & $95 \% \mathrm{Cl}^{\mathrm{a}}$ \\
\hline Baseline & & 1 Day & 17.41 & & 0.88 & $<0.0001$ & 14.78 to 20.03 \\
\hline & & 2 Weeks & 15.29 & & 0.88 & $<0.0001$ & 12.67 to 17.92 \\
\hline & & 1 Month & 14.65 & & 0.88 & $<0.0001$ & 12.02 to 17.28 \\
\hline & & 3 Months & 13.79 & & 0.88 & $<0.0001$ & 11.16 to 16.42 \\
\hline & & 6 Months & 12.59 & & 0.94 & $<0.0001$ & 9.78 to 15.40 \\
\hline 1 Day & & Baseline & -17.41 & & 0.88 & $<0.0001$ & -20.03 to -14.78 \\
\hline & & 2 Weeks & -2.11 & & 0.88 & 0.27 & -4.73 to 0.51 \\
\hline & & 1 Month & -2.75 & & 0.88 & 0.0314 & -5.38 to -0.12 \\
\hline & & 3 Months & -3.61 & & 0.88 & 0.0009 & -6.24 to -0.98 \\
\hline & & 6 Months & -4.81 & & 0.94 & $<0.0001$ & -7.62 to -2.00 \\
\hline
\end{tabular}


Table 4 Two-way ANOVA with tests of between-subjects effects for all variables tested, estimated marginal means and pairwise comparison for the IOP dependent variable and intergroup t-test comparison between Preserflo Microshunt with MMC $0.2 \mathrm{mg} / \mathrm{ml}$ and Preserflo Microshunt with MMC $0.2 \mathrm{mg} / \mathrm{ml}$ and Ologen implantation (Continued)

\begin{tabular}{|c|c|c|c|c|c|}
\hline \multicolumn{6}{|l|}{ Two-way ANOVA } \\
\hline Pairwise comparisons (IOP dependent) & & Mean difference & Std. Error & $P^{a}$ & $95 \% \mathrm{Cl}^{\mathrm{a}}$ \\
\hline \multirow[t]{5}{*}{2 Weeks } & Baseline & -15.29 & 0.88 & $<0.0001$ & -17.92 to -12.67 \\
\hline & 1 Day & 2.11 & 0.88 & 0.27 & -0.51 to 4.73 \\
\hline & 1 Month & -0.64 & 0.88 & 1.00 & -3.27 to 1.98 \\
\hline & 3 Months & -1.50 & 0.88 & 1.00 & -4.13 to 1.12 \\
\hline & 6 Months & -2.70 & 0.94 & 0.07 & -5.51 to 0.10 \\
\hline \multirow[t]{5}{*}{1 Month } & Baseline & -14.65 & 0.88 & $<0.0001$ & -17.28 to -12.02 \\
\hline & 1 Day & 2.75 & 0.88 & 0.0314 & 0.12 to 5.38 \\
\hline & 2 Weeks & 0.64 & 0.88 & 1.00 & -1.98 to 3.27 \\
\hline & 3 Months & -0.85 & 0.88 & 1.00 & -3.48 to 1.77 \\
\hline & 6 Months & -2.05 & 0.94 & 0.46 & -4.86 to 0.75 \\
\hline \multirow[t]{5}{*}{3 Months } & Baseline & -13.79 & 0.88 & $<0.0001$ & -16.42 to -11.16 \\
\hline & 1 Day & 3.61 & 0.88 & 0.0009 & 0.98 to 6.24 \\
\hline & 2 Weeks & 1.50 & 0.88 & 1.00 & -1.12 to 4.13 \\
\hline & 1 Month & 0.85 & 0.88 & 1.00 & -1.77 to 3.48 \\
\hline & 6 Months & -1.20 & 0.94 & 1.00 & -4.01 to 1.61 \\
\hline \multirow[t]{5}{*}{6 Months } & Baseline & -12.59 & 0.94 & $<0.0001$ & -15.40 to -9.78 \\
\hline & 1 Day & 4.81 & 0.94 & $<0.0001$ & 2.00 to 7.62 \\
\hline & 2 Week & 2.70 & 0.94 & 0.07 & -0.10 to 5.51 \\
\hline & 1 Month & 2.05 & 0.94 & 0.46 & -0.75 to 4.86 \\
\hline & 3 Months & 1.20 & 0.94 & 1.00 & -1.61 to 4.01 \\
\hline
\end{tabular}

\footnotetext{
a Bonferroni corrected

${ }^{\mathrm{b}}$ Computed using alpha $=0.05$

Group A: Preserflo Microshunt with MMC $0.2 \mathrm{mg} / \mathrm{ml}$

Group B: Preserflo Microshunt with MMC $0.2 \mathrm{mg} / \mathrm{ml}$ and Ologen

SD: standard deviation; DF: degrees of freedom; Eta: eta coefficient (An eta coefficient test is a method for determining the strength of association between a categorical variable)
}

performed at 6 months in group B $(12 \%)$ in comparison to one in group A (4\%). Regarding bleb fibrosis, both groups shared similar characteristics in terms of age, prior surgery status and ethnicity (Table 5). All eyes were conjunctiva surgery naive prior to Preserflo Microshunt implantation and had only undergone cataract extraction. During revision surgeries, the conjunctiva, Tenon's capsule, sclera, and the Preserflo Microshunt were examined. After removal of fibrosis, the Preserflo Microshunt was checked for flow. If flow was inadequate, the tube was removed from the scleral pocket and flushed with BSS. Upon successful irrigation and adequate flow, the tube was returned back into position following scleral treatment with $0.2 \mathrm{mg} / \mathrm{ml} \mathrm{MMC} \mathrm{for} 3 \mathrm{~min}$.

\section{Discussion}

Trabeculectomy is still regarded by many surgeons as the gold standard in surgical treatment of POAG patients $[15-17,24]$. Nonetheless, its high complication rate and demanding postoperative management have caused surgeons to seek alternative surgical approaches [25]. Such approaches include the XEN Glaucoma Gel Microstent implant (XENGGM, Allergan Plc., Parsippany, New Jersey), an ab interno alternative approach to trabeculectomy, which has gained vast popularity in recent years [26, 27]. XEN reduces IOP significantly and is designed to avoid postoperative hypotony which is a major advantage over the traditional trabeculectomy approach. Studies have however shown that in the long term, additional revision surgeries due to bleb fibrosis ranging from 32 to $37.7 \%$ are required to maintain a sufficient low IOP without medication [28, 29]. The Preserflo Microshunt is a relatively novel ab externo alternative to trabeculectomy. There are a few known studies to date following Preserflo Microshunt implantation outcomes [12, 30, 31, 32, 33]. Pinchuck et al. [12] first reported on a cohort of 23 successful Preserflo Microshunt implantations in 23 


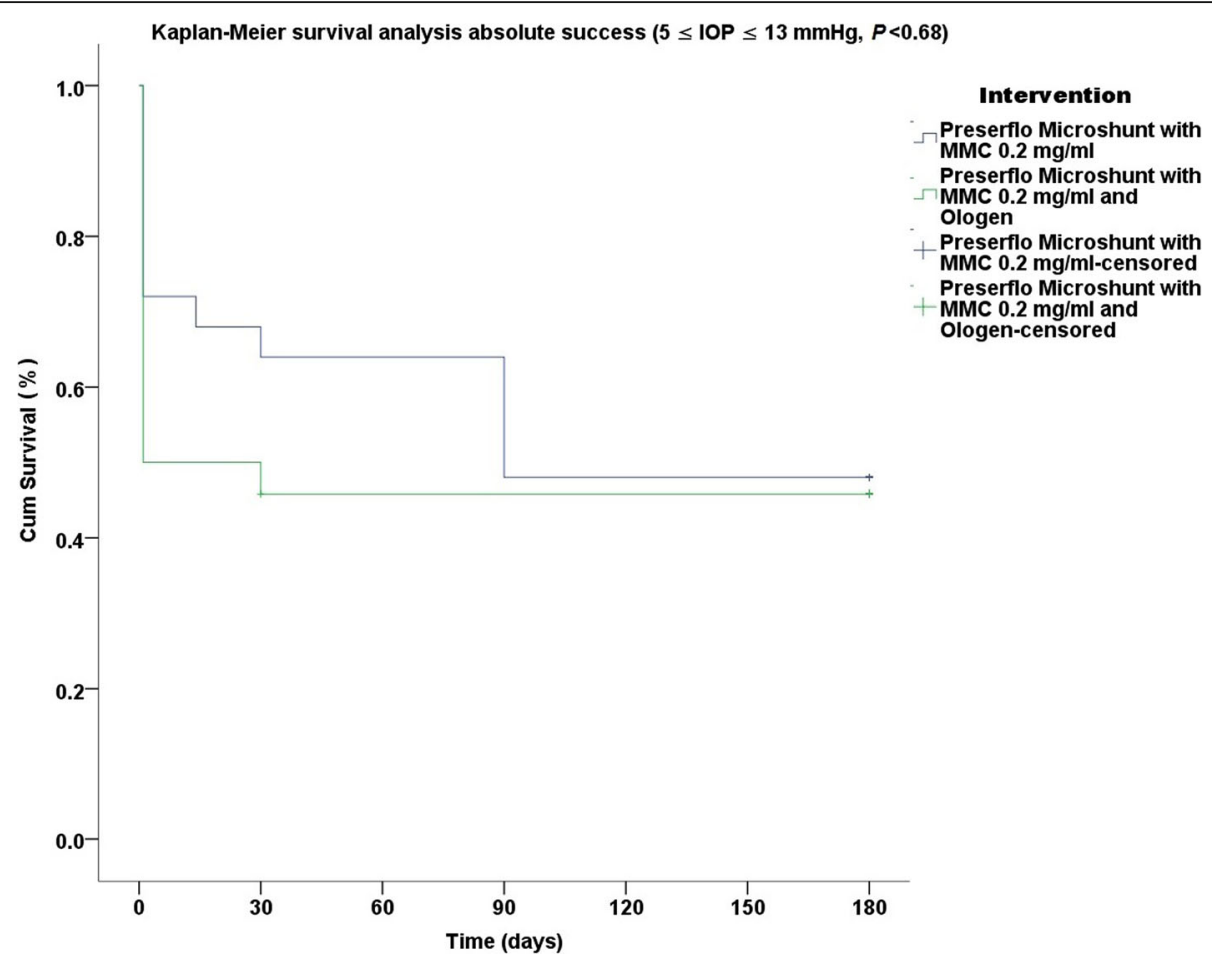

Fig. 2 Log-rank, Kaplan-Meier survival analysis of absolute success rate between Preserflo Microshunt with MMC $0.2 \mathrm{mg} / \mathrm{ml}$ and Preserflo Microshunt with MMC $0.2 \mathrm{mg} / \mathrm{ml}$ and Ologen implantation $(5 \leq 1 \mathrm{OP} \leq 13 \mathrm{mmHg}, P<0.68$ )

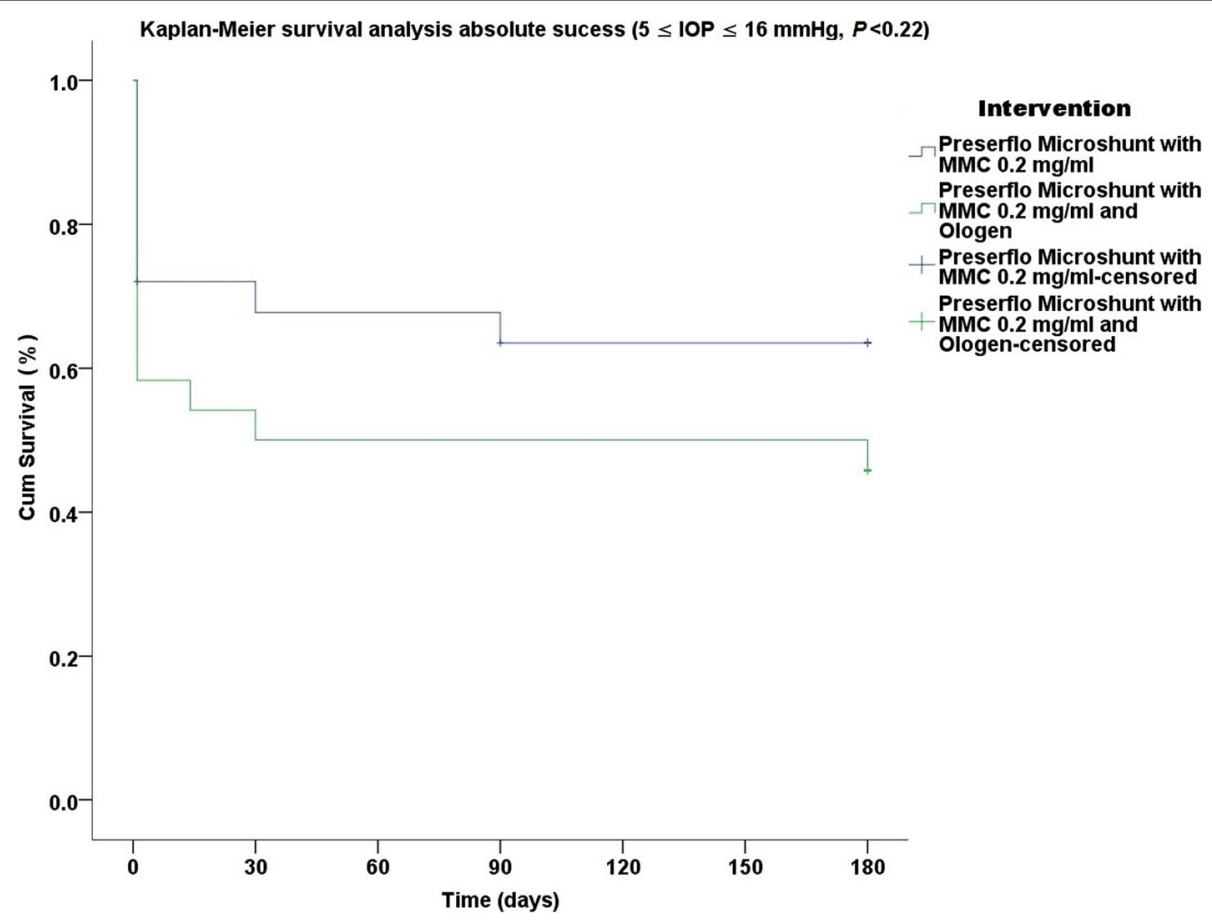

Fig. 3 Log-rank, Kaplan-Meier survival analysis of absolute success rate between Preserflo Microshunt with MMC $0.2 \mathrm{mg} / \mathrm{ml}$ and Preserflo Microshunt with MMC $0.2 \mathrm{mg} / \mathrm{ml}$ and Ologen implantation $(5 \leq \mathrm{IOP} \leq 16 \mathrm{mmHg}, P<0.22)$ 


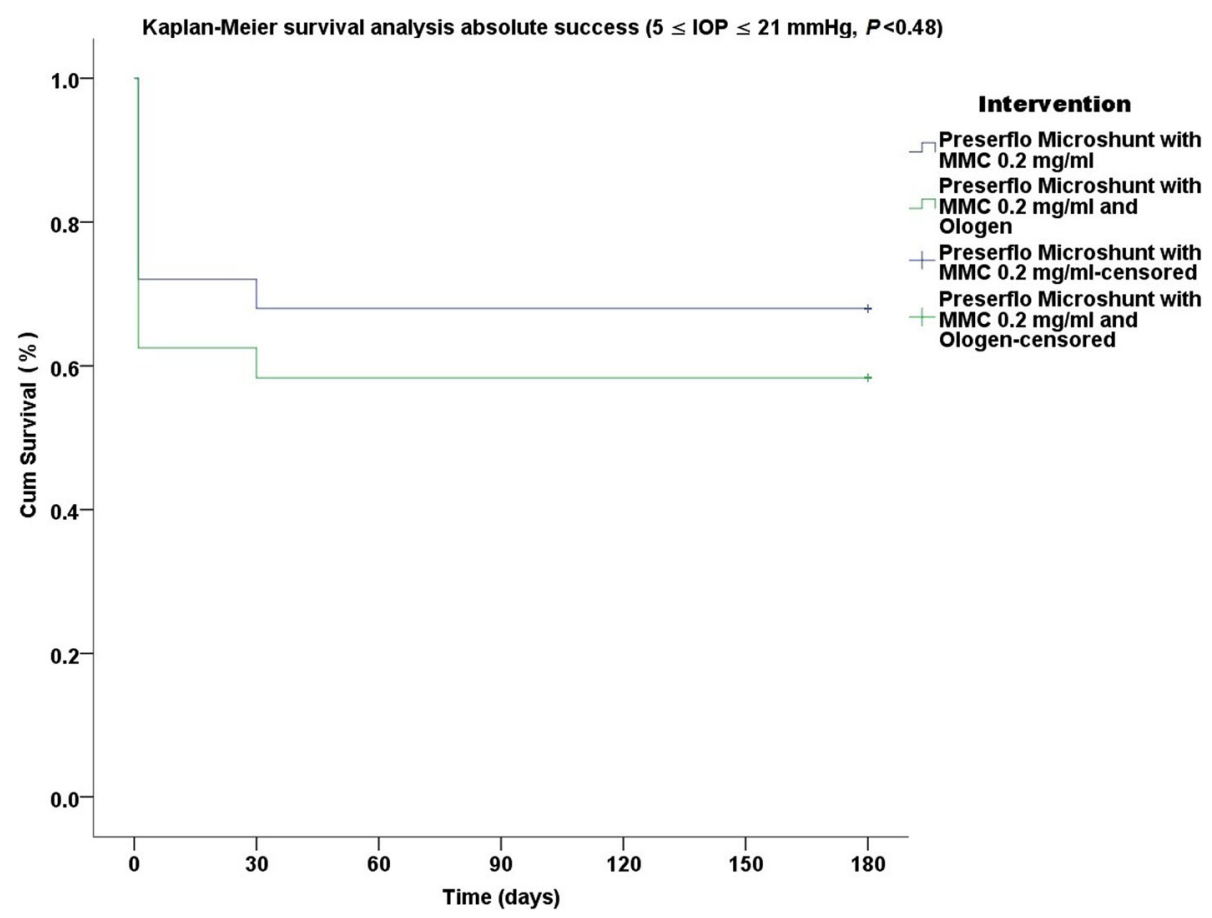

Fig. 4 Log-rank, Kaplan-Meier survival analysis of absolute success rate between Preserflo Microshunt with MMC $0.2 \mathrm{mg} / \mathrm{ml}$ and Preserflo Microshunt with MMC $0.2 \mathrm{mg} / \mathrm{ml}$ and Ologen implantation $(5 \leq \mathrm{IOP} \leq 21 \mathrm{mmHg}, P<0.48)$

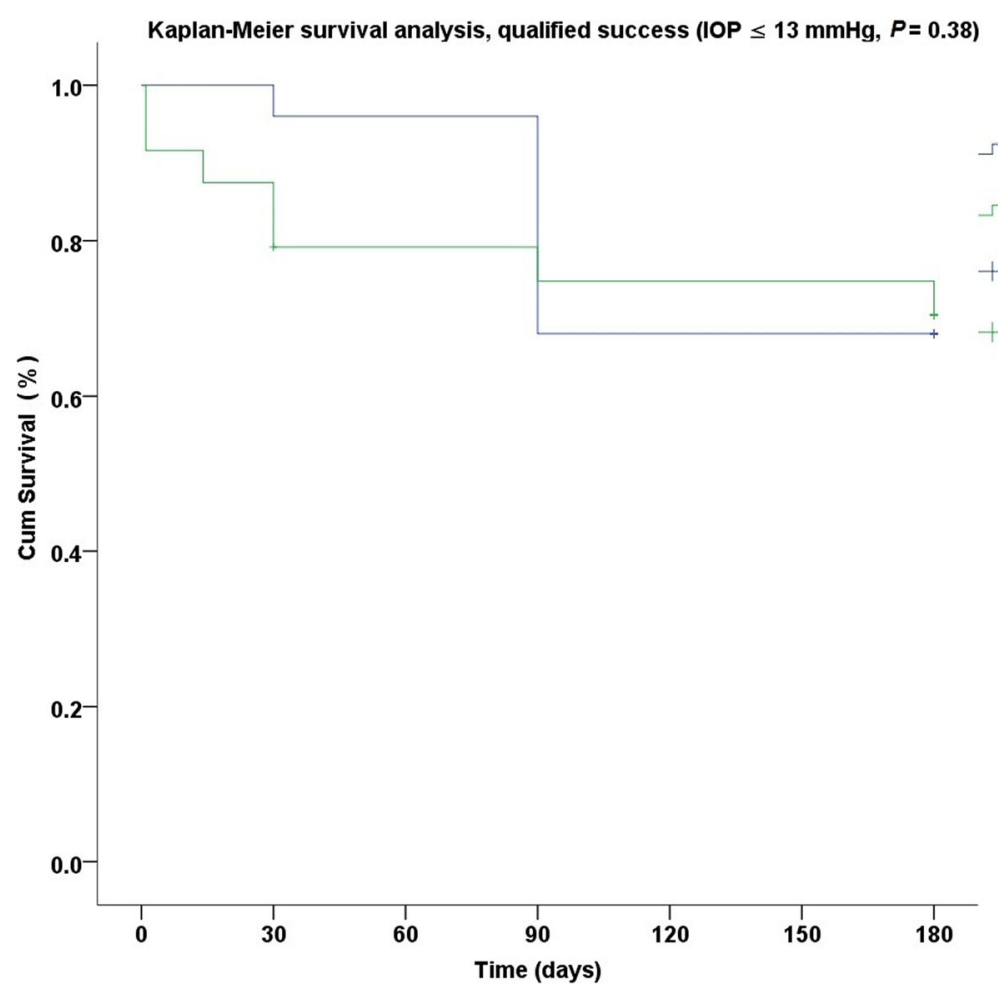

Fig. 5 Log-rank, Kaplan-Meier survival analysis of qualified success rate between Preserflo Microshunt with MMC $0.2 \mathrm{mg} / \mathrm{ml}$ and Preserflo Microshunt with MMC $0.2 \mathrm{mg} / \mathrm{ml}$ and Ologen implantation (IOP $\leq 13 \mathrm{mmHg}$ with or without medication, $P=0.38$ ) 


\section{Kaplan-Meier survival analysis, qualified success (IOP $\leq 16 \mathrm{mmHg}, P<0.31$ )}

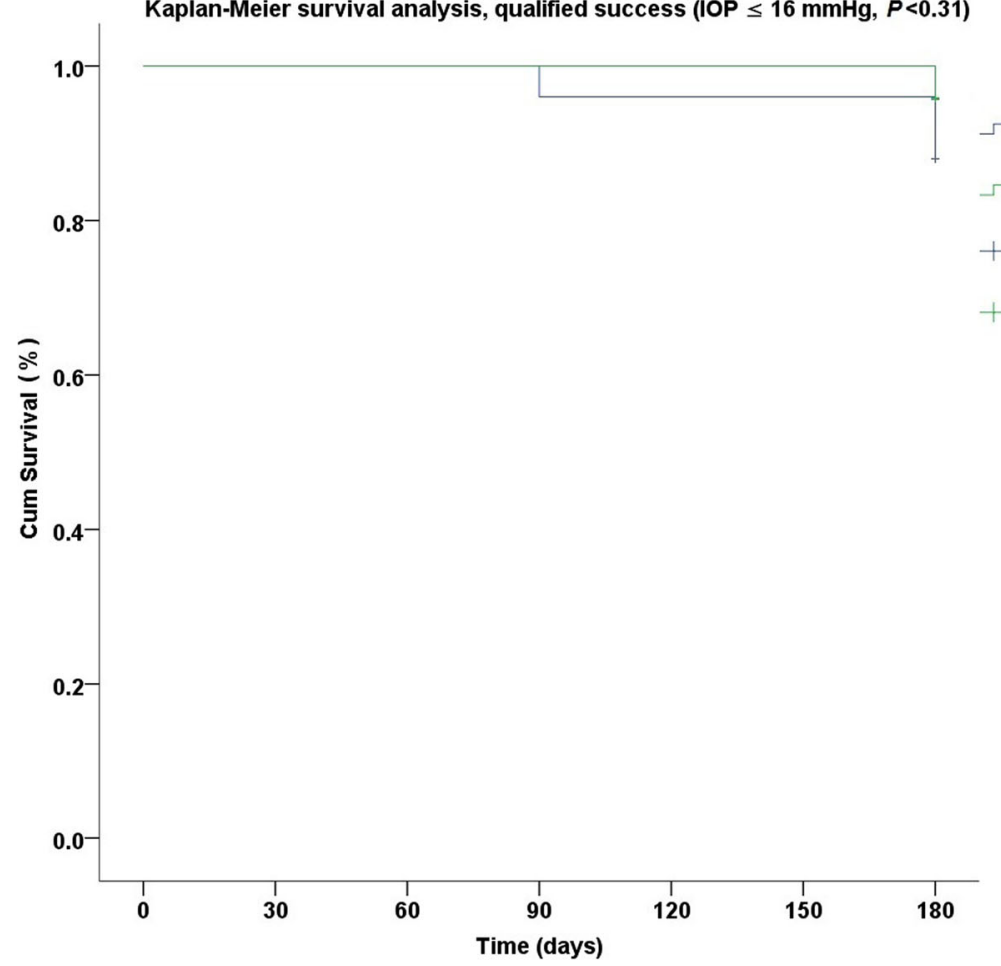

Intervention

Preserflo Microshunt with

MMC $0.2 \mathrm{mg} / \mathrm{ml}$

Preserflo Microshunt with

MMC $0.2 \mathrm{mg} / \mathrm{ml}$ and

Ologen

Preserflo Microshunt with

MMC $0.2 \mathrm{mg} / \mathrm{ml}$-censored

Preserflo Microshunt with

MMC $0.2 \mathrm{mg} / \mathrm{ml}$ and

ologen-censored

Fig. 6 Log-rank, Kaplan-Meier survival analysis of qualified success rate between Preserflo Microshunt with MMC $0.2 \mathrm{mg} / \mathrm{ml}$ and Preserflo Microshunt with MMC $0.2 \mathrm{mg} / \mathrm{ml}$ and Ologen implantation (IOP $\leq 16 \mathrm{mmHg}$ with or without medication, $P<0.31$ )

consecutive eyes. In this study, the design and features of the device as well as the steps of the surgical procedure were thoroughly described and reported. The minimal hypotony results in combination with the insignificant bleb inflammation and encapsulation results as well as the effectiveness of the procedure in lowering the IOP postoperatively provided an alternative to primary trabeculectomy. In this study, the IOP was lowered by 50 to $55 \%$ reaching a level below $14 \mathrm{mmHg}$ in over $80 \%$ of patients. Similar results were reported by Riss et al. in a retrospective two-center, two-surgeon study with one-year follow-up [30]. In that observational study, the primary goal was to demonstrate the significance of different MMC concentrations, 0.4 versus $0.2 \mathrm{mg} /$ $\mathrm{ml}$, and different placement of an MMC sponge in anatomical locations, limbus versus deep in the pocket, in terms of IOP and medication reduction in three distinct groups. They reported a sufficient IOP and medication reduction postoperatively ranging from 38 to $55 \%$ and 72 to $85 \%$ at 12 months, respectively. The effectiveness in IOP and medication reduction following a Preserflo Microshunt implantation has been well documented in the recent years [30, 31, 32, 33]. In our current study, we found comparable results in terms of mean IOP reduction at 6 months from $23.52 \pm 5.78$ to $11.56 \pm 3.08 \mathrm{mmHg}$ and from $26.04 \pm 8.76$ to $11.75 \pm 3.37 \mathrm{mmHg}$ in groups A and B with a $49.06 \%$ mean IOP reduction in group $\mathrm{A}$ and $53.01 \%$ mean IOP reduction in group $B$. The mean medication reduction in our study was also similar and comparable to other studies results $[12,30,31,32,33]$ with medication reduction in group A ranging from $2.52 \pm 0.91$ to $0.04 \pm 0.20$ medications $(98.02 \%$ reduction, median reduction of 2.5 medications) and in group B from $2.58 \pm 0.82$ to $0.16 \pm 0.81$ medications $(94.44 \%$ reduction, median reduction of 2.5 medications) by the 6th month. In terms of absolute and qualified success, different criteria were used in all the studies so an accurate comparison is not possible. Battle et al. [32] defined qualified success as having an IOP of less than $14 \mathrm{mmHg}$ and a minimum IOP reduction of $20 \%$ (IOP $\leq 14 \mathrm{mmHg}$ and IOP reduction $\geq 20 \%$ ). Success rate reported was $100 \%, 91 \%$, and $95 \%$ for the 1st, 2nd and 3rd year, respectively. Schlenker et al. [33] defined absolute success as an IOP greater than 6 and less than $17 \mathrm{mmHg}$ without hypotony $(6<\mathrm{IOP}<17 \mathrm{mmHg})$ and qualified as the same range with glaucoma medications with at least a $20 \%$ IOP reduction. Complete success was achieved in $76.9 \%$ of eyes, qualified success in $92.5 \%$. Complete success 


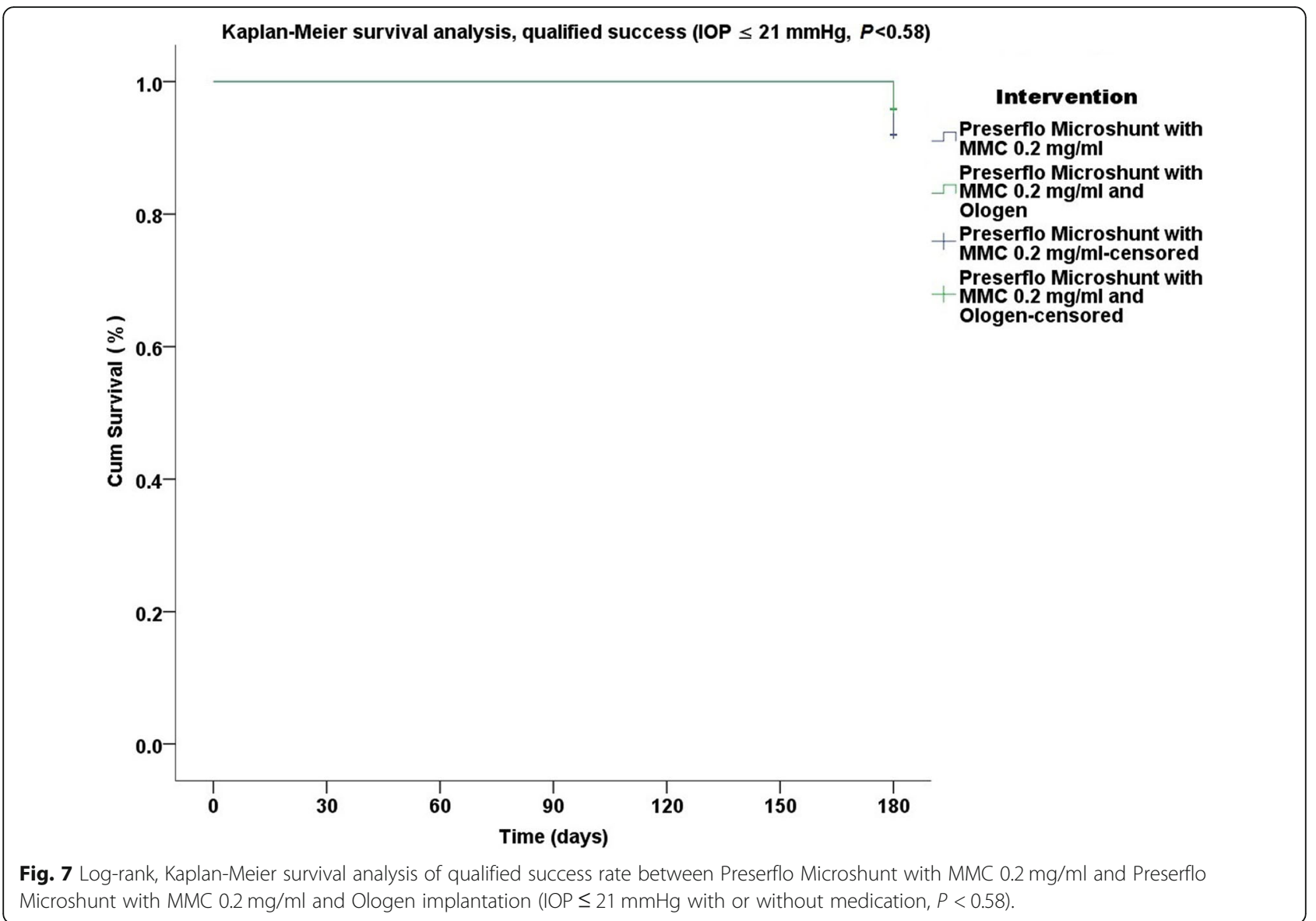

Microshunt with MMC $0.2 \mathrm{mg} / \mathrm{ml}$ and Ologen implantation (IOP $\leq 21 \mathrm{mmHg}$ with or without medication, $P<0.58$ ).

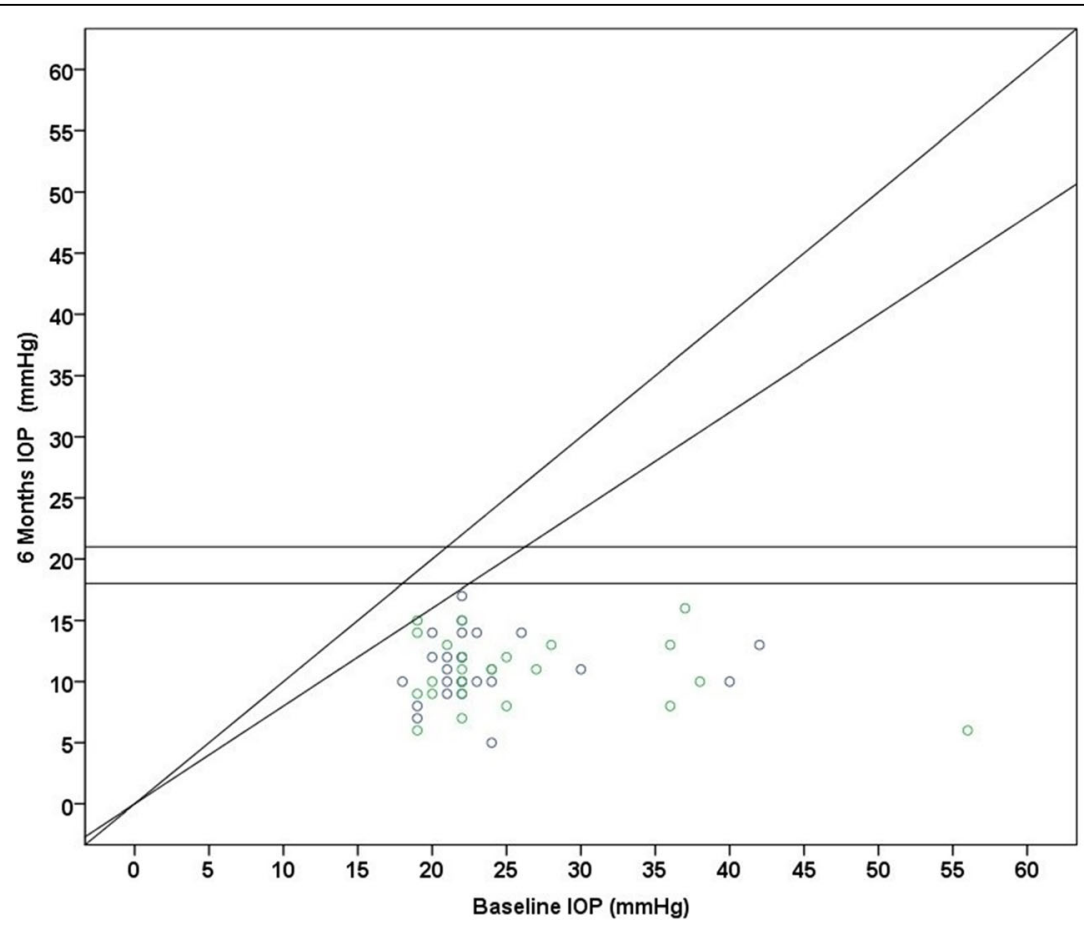

$20 \%$ IOP reduction

Preserflo Microshunt

with MMc $0.2 \mathrm{mg} / \mathrm{ml}$

with $\mathrm{MMC} 0.2 \mathrm{mg} / \mathrm{ml}$ and Ologen

Fig. 8 Scatterplot analysis of pressure control of effect data of all eyes $(n=50)$ between baseline and 6 months intraocular pressure (IOP) values. 


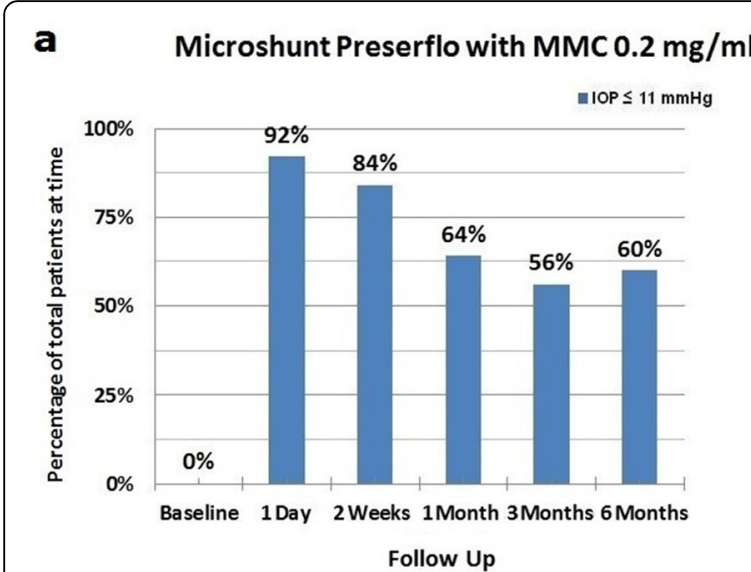

b

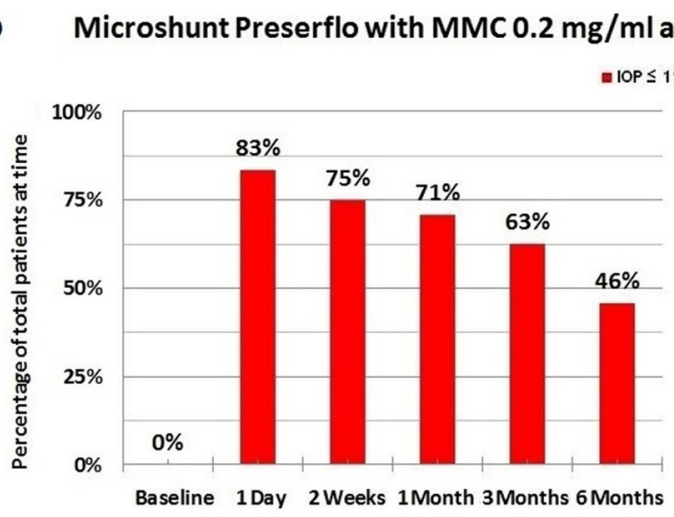

Follow Up

\section{d}

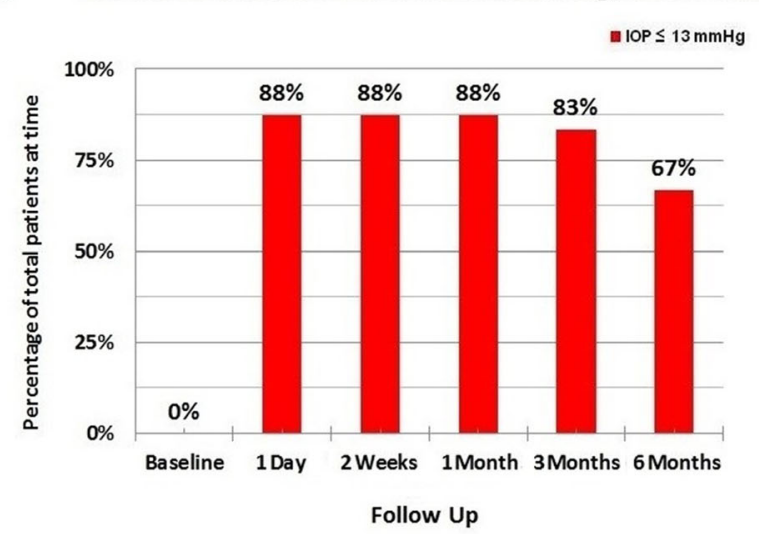

e Microshunt Preserflo with MMC $0.2 \mathrm{mg} / \mathrm{ml}$

f
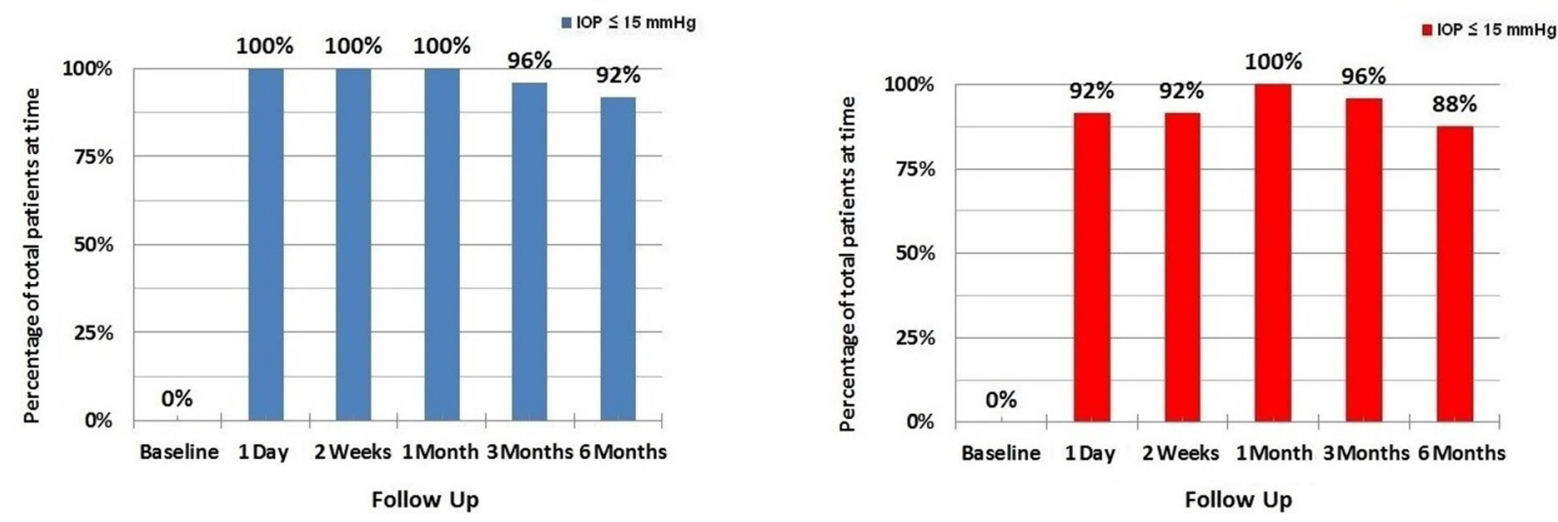

Fig. 9 Cumulative intraocular pressure (IOP) results per group (Preserflo Microshunt with MMC $0.2 \mathrm{mg} / \mathrm{ml}$ and Preserflo Microshunt with MMC 0.2 $\mathrm{mg} / \mathrm{ml}$ and Ologen implantation) in percentage for IOP readings less than or equal to (a, b) $11 \mathrm{mmHg}$, (c, d) $13 \mathrm{mmHg}$ and (e, f) $15 \mathrm{mmHg}$

was $75.6 \%$ for an upper IOP cut-off of $14 \mathrm{mmHg}$ and $76.9 \%$ for $21 \mathrm{mmHg}$, and qualified success was 91.9 and $92.5 \%$. Needling rate was $8.5 \%$. Durr et al. similarly to Schlenker et al. [33] defined failure as an IOP less than $6 \mathrm{mmHg}$ with vision loss, greater than $17 \mathrm{mmHg}$ or less of $20 \%$ reduction in IOP without medication. Secondary outcomes included thresholds of 6 to $14 \mathrm{mmHg}$ and 6 to $21 \mathrm{mmHg}$ for both complete (no medication) and qualified (with medication) success as well as qualified success for thresholds of 6 to $17 \mathrm{mmHg}$. A $61.0 \%$ complete success and $79.7 \%$ qualified success rate was reported. 
Table 5 Bleb failure risk factors and failure results per group

\begin{tabular}{lll}
\hline Demographics & Group A & Group B \\
\hline No. of eyes operated total & 25 & 25 \\
Prior ocular surgeries & Phacoemulsification & Phacoemulsification \\
Ethnicity of patients & Caucasian & Caucasian \\
No. of female patients & 18 & 15 \\
Age (years) & $77.58 \pm 7.62$ & $75.64 \pm 12.61$ \\
No. of male patients & 7 & 10 \\
Bleb revision surgery at 6 & $1(4 \%)$ & $3(12 \%)$ \\
months (failure rate) & &
\end{tabular}

MMC mitomycin C

Group A: Preserflo Microshunt with MMC $0.2 \mathrm{mg} / \mathrm{ml}$

Group B: Preserflo Microshunt with MMC $0.2 \mathrm{mg} / \mathrm{ml}$ and Ologen

Needling was performed in $11.8 \%$ of eyes. We reported comparable results with absolute success rate in group A reaching a) $48 \%$, b) $64 \%$ and c) $68 \%$ in comparison to group B a) $45.8 \%$, b) $45.8 \%$ and c) $58.3 \%$ at 6 months (Figs. 2, 3 and 4 respectively). Our qualified success rate in group A was a) $68 \%$, b) $88 \%$ and c) $92 \%$ in comparison to group B a) $70.8 \%$, b) $91.8 \%$ and c) $95.8 \%$ at 6 months (Figs. 5, 6 and 7 respectively). Four revision surgeries due to bleb fibrosis were performed at 6 months and were considered failures (8\%). Three revision surgeries were performed at 6 months in group B (12\%) in comparison to one in group A (4\%). In our cohort, a concentration of MMC $0.2 \mathrm{mg} / \mathrm{ml}$ was used in all cases with placement of the soaked sponges posteriorly or deep in the pocket for $3 \mathrm{~min}$. We found that the results of Riss et al. in their group C of $38 \%$ mean IOP reduction with $\mathrm{MMC} 0.4 \mathrm{mg} / \mathrm{ml}$ scleral treatment deep in the pocket at one-year follow-up were not comparable with our results. In the studies by Schlenker and Durr, [32, 33] different MMC dose of 0.2 versus $0.4-0.5 \mathrm{mg} / \mathrm{ml}$ were used in POAG versus secondary open angle glaucoma (SOAG). Our cohort included only POAG eyes with conjunctival naive patients. Other differing factors between the studies of Battle, Durr and Schlenker [31, 32, 33] and ours, were the cohorts' ethnic group, primarily AfroCaribbean versus Caucasian, combined surgery with phacoemulsification versus standalone procedure, different MMC dose and the OCM implantation. Regarding the possible benefit provided from OCM implantation we did not find any statistical differences in terms of IOP reduction between the groups at 6 months. In group B, we performed two additional revision surgeries in comparison to group A, although both groups shared almost identical characteristics in terms of bleb failure risk (Table 5). Our current results showed no statistical difference between groups A and B. Similar results were reported in the survival analysis (Figs. 2 and 3 ) and in the scatterplot analysis (Fig. 8) demonstrating equal IOP reduction among the groups. Our cumulative IOP results also did not demonstrate any statistical differences between groups A and B at 6 months (Fig. 9). In our opinion, an OCM additional implantation could have been of benefit in a cohort with mixed ethnic origin patients other than Caucasian, prior glaucoma surgery and those on long term glaucoma medication.

\section{Conclusion}

We were able to reproduce similar results to preexisting studies $[11,29,30,31,32,33]$. We did not report any adverse or severe sight-threatening complications and our revision surgery rate was $8 \%$. Our greatest limitation is only having 6 months of data, the retrospective nature of the study and failing the assumption of homogeneity of variance. We advise caution when interpreting our results in terms of OCM evaluation and IOP or medication reduction as well as the current revision rates presented. Longterm follow-up is required to evaluate any benefit in terms of wound modulation of an additional OCM implantation following Preserflo Microshunt surgery.

\begin{abstract}
Acknowledgements
We thank Dr. Clifford Lamptey GP, specialist in the republic of Ireland, MB, BCh BAO LRPC\&SI NUI (Hons), MRCS, MRCPsych MICGP for language editing and proof reading.
\end{abstract}

\section{Authors' contributions}

IV: Conceptualization, original draft, surgery, methodology, software, statistical analysis, writing, review and editing, supervision; SF: Surgery, methodology, statistical analysis, writing, review and editing; GP: Editing, surgery, visualization; KK: Statistical analysis, surgery; MB: Validation, methodology; ZG: Validation, methodology; MK: Conceptualization, methodology, surgery, general supervision. All authors read and approved the final manuscript.

\section{Funding}

This research received no specific grant from any funding agency in the public, commercial, or not-for-profit sectors.

\section{Availability of data and materials \\ Not applicable.}

\section{Declaration}

Ethics approval and consent to participate

The institution's ethical committee approved this retrospective case series study (01/2019/004_OPH_Preserflo MMC).

\section{Consent for publication}

Not applicable.

\section{Competing interests}

The authors declare that they have no competing interests.

\section{Author details}

'Department of Ophthalmology, St. Johannes Academic Hospital Dortmund, Dortmund, Germany. ${ }^{2}$ Ophthalmica Eye Institute, Thessaloniki, Greece.

${ }^{3}$ Department of Ophthalmology, University Hospital Basel, Basel, Switzerland. 
Received: 7 February 2021 Accepted: 21 July 2021

Published online: 03 September 2021

\section{References}

1. Quigley HA. Number of people with glaucoma worldwide. Br J Ophthalmol. 1996;80(5):389-93.

2. Tham YC, Li X, Wong TY, Quigley HA, Aung T, Cheng CY. Global prevalence of glaucoma and projections of glaucoma burden through 2040: a systematic review and meta-analysis. Ophthalmology. 2014;121(11):208190

3. Heijl A, Leske MC, Bengtsson B, Hyman L, Bengtsson B, Hussein M, et al. Reduction of intraocular pressure and glaucoma progression: results from the Early Manifest Glaucoma Trial. Arch Ophthalmol. 2002;120(10):1268-79.

4. Kass MA, Heuer DK, Higginbotham EJ, Johnson CA, Keltner JL, Miller JP, et al. The Ocular Hypertension Treatment Study: a randomized trial determines that topical ocular hypotensive medication delays or prevents the onset of primary open-angle glaucoma. Arch Ophthalmol. 2002;120(6): 701-13; discussion 829-30.

5. Lichter PR, Musch DC, Gillespie BW, Guire KE, Janz NK, Wren PA, et al. Interim clinical outcomes in the Collaborative Initial Glaucoma Treatment Study comparing initial treatment randomized to medications or surgery. Ophthalmology. 2001;108(11):1943-53.

6. Sawchyn AK, Slabaugh MA. Innovations and adaptations in trabeculectomy. Curr Opin Ophthalmol. 2016a;27(2):158-63.

7. Lavia C, Dallorto L, Maule M, Ceccarelli M, Fea AM. Minimally-invasive glaucoma surgeries (MIGS) for open angle glaucoma: a systematic review and meta-analysis. PLoS One. 2017;12(8):e0183142.

8. King AJ, Shah A, Nikita E, Hu K, Mulvaney CA, Stead R, et al. Subconjunctival draining minimally-invasive glaucoma devices for medically uncontrolled glaucoma. Cochrane Database Syst Rev. 2018;12(12):CD012742.

9. Cutolo CA, lester M, Bagnis A, Bonzano C, Negri L, Olivari S, et al. Early postoperative intraocular pressure is associated with better pressure control after XEN implantation. J Glaucoma. 2020;29(6):456-60.

10. Mansouri K, Guidotti J, Rao HL, Ouabas a, D'Alessandro E, Roy S, et al. prospective evaluation of standalone XEN gel implant and combined phacoemulsification-XEN gel implant surgery: 1-year results. J Glaucoma. 2018:27(2):140-7.

11. Pinchuk L, Riss I, Batlle JF, Kato YP, Martin JB, Arrieta E, et al. The use of poly (styrene-block-isobutylene-block-styrene) as a microshunt to treat glaucoma. Regen Biomater. 2016;3(2):137-42.

12. Pinchuk L, Riss I, Batlle JF, Kato YP, Martin JB, Arrieta E, et al. The development of a micro-shunt made from poly (styrene-block-isobutyleneblock-styrene) to treat glaucoma. J Biomed Mater Res B Appl Biomater. 2017:105(1):211-21.

13. Pinchuk L, Wilson GJ, Barry JJ, Schoephoerster RT, Parel JM, Kennedy JP. Medical applications of poly (styrene-block-isobutylene-block-styrene) ("SIBS"). Biomaterials. 2008;29(4):448-60.

14. Cillino S, Di Pace F, Cillino G, Casuccio A. Biodegradable collagen matrix implant vs mitomycin-C as an adjuvant in trabeculectomy: a 24-month, randomized clinical trial. Eye (Lond). 2011;25(12):1598-606.

15. Fili S, Seddig S, Kohlhaas M. Long-term results after trabeculectomy combined with mitomycin C and Ologen implant. Klin Monatsbl Augenheilkd. 2019;236(9):1107-14.

16. Papaconstantinou D, Georgalas I, Karmiris E, Diagourtas A, Koutsandrea C, Ladas I, et al. Trabeculectomy with OloGen versus trabeculectomy for the treatment of glaucoma: a pilot study. Acta Ophthalmol. 2010;88(1):80-5.

17. Rho S, Sung Y, Ma KT, Rho SH, Kim CY. Bleb analysis and short-term results of biodegradable collagen matrix-augmented Ahmed glaucoma valve implantation: 6-month follow-up. Invest Ophthalmol Vis Sci. 2015;56(10): 5896-903.

18. Chen $X$, Yuan F. Ologen implantation versus conjunctival autograft transplantation for treatment of pterygium. J Ophthalmol. 2018;2018: 1617520.

19. Picht G, Grehn F. Classification of filtering blebs in trabeculectomy: biomicroscopy and functionality. Curr Opin Ophthalmol. 1998;9(2):2-8

20. Picht G, Grehn F. Development of the filtering bleb after trabeculectomy. Classification, histopathology, wound healing process. Ophthalmologe. 1998;95(5):W380-7.

21. Klink J, Schmitz B, Lieb WE, Klink T, Grein HJ, Sold-Darseff J, et al. Filtering bleb function after clear cornea phacoemulsification: a prospective study. $\mathrm{Br}$ J Ophthalmol. 2005;89(5):597-601.
22. Hodapp E, Parrish RK II, Anderson DR. Clinical decisions in glaucoma. St Louis: The CV Mosby Co; 1993. p. 52-61.

23. Sawchyn AK, Slabaugh MA. Innovations and adaptations in trabeculectomy. Curr Opin Ophthalmol. 2016;27(2):158-63.

24. Zahid S, Musch DC, Niziol LM. Lichter PR; Collaborative Initial Glaucoma Treatment Study Group. Risk of endophthalmitis and other long-term complications of trabeculectomy in the Collaborative Initial Glaucoma Treatment Study (CIGTS). Am J Ophthalmol. 2013;155(4):674-80.

25. Lenzhofer M, Kersten-Gomez I, Sheybani A, Gulamhusein H, Strohmaier C, Hohensinn $M$, et al. Four-year results of a minimally invasive transscleral glaucoma gel stent implantation in a prospective multi-centre study. Clin Exp Ophthalmol. 2019;47(5):581-7.

26. Gillmann K, Bravetti GE, Mermoud A, Rao HL, Mansouri K. XEN Gel Stent in pseudoexfoliative glaucoma: 2-year results of a prospective evaluation. J Glaucoma. 2019:28(8):676-84

27. Mansouri K, Gillmann K, Rao HL, Guidotti J, Mermoud A. Prospective evaluation of XEN Gel implant in eyes with pseudoexfoliative glaucoma. J Glaucoma. 2018;27(10):869-73.

28. Buffault J, Baudouin C, Labbé A. XEN ${ }^{\circledast}$ Gel Stent for management of chronic open angle glaucoma: a review of the literature. J Fr Ophtalmol. 2019;42(4): 391-403.

29. Riss I, Batlle J, Pinchuk L, Kato YP, Weber BA, Parel JM. One-year results on the safety and efficacy of the InnFocus MicroShunt ${ }^{\mathrm{TM}}$ depending on placement and concentration of mitomycin C. J Fr Ophtalmol. 2015;38(9): 855-60

30. Sadruddin O, Pinchuk L, Angeles R, Palmberg P. Ab externo implantation of the MicroShunt, a poly (styrene-block-isobutylene-block-styrene) surgical device for the treatment of primary open-angle glaucoma: a review. Eye Vis (Lond). 2019;6:36.

31. Batlle JF, Fantes F, Riss I, Pinchuk L, Alburquerque R, Kato YP, et al. Threeyear follow-up of a novel aqueous humor microshunt. J Glaucoma. 2016; 25(2):e58-65.

32. Schlenker MB, Durr GM, Michaelov E, Ahmed IIK. Intermediate outcomes of a novel standalone ab externo SIBS microshunt with mitomycin C. Am J Ophthalmol. 2020;215:141-53.

33. Durr GM, Schlenker MB, Samet S, Ahmed IIK. One-year outcomes of standalone ab externo SIBS microshunt implantation in refractory glaucoma. $\mathrm{Br}$ J Ophthalmol. 2020 Oct;23 bjophthalmol-2020-317299.

\section{Ready to submit your research? Choose BMC and benefit from:}

- fast, convenient online submission

- thorough peer review by experienced researchers in your field

- rapid publication on acceptance

- support for research data, including large and complex data types

- gold Open Access which fosters wider collaboration and increased citations

- maximum visibility for your research: over $100 \mathrm{M}$ website views per year

At $\mathrm{BMC}$, research is always in progress.

Learn more biomedcentral.com/submissions 